\title{
3 Research Square

\section{Pharmacokinetics and intrapulmonary concentrations of high-dose tigecycline in critically ill patients with severe infections}

Gennaro De Pascale ( $\sim$ gennaro.depascalemd@gmail.com )

Roma-Universita' Cattolica del Sacro Cuo https://orcid.org/0000-0002-8255-0676

Lucia Lisi

Fondazione Policlinico A. Gemelli IRCCS

Gabriella Maria Pia Ciotti

Fondazione Policlinico A. Gemelli IRCCS

Maria Sole Vallecoccia

Fondazione Policlinico A. Gemelli IRCCS

Salvatore Lucio Cutuli

Fondazione Policlinico A. Gemelli IRCCS

Laura Cascarano

Fondazione Policlinico A. Gemelli IRCCS

Camilla Gelormini

Fondazione Policlinico A. Gemelli IRCCS

Giuseppe Bello

Fondazione Policlinico A. Gemelli IRCCS

Luca Montini

Fondazione Policlinico A. Gemelli IRCCS

Simone Carelli

Fondazione Policlinico A. Gemelli IRCCS

Valentina Di Gravio

Fondazione Policlinico A. Gemelli IRCCS

Mario Tumbarello

Fondazione Policlinico A. Gemelli IRCCS

Maurizio Sanguinetti

Policlinico Universitario Agostino Gemelli IRCCS

Pierluigi Navarra

Policlinico Universitario Agostino Gemelli IRCCS

Massimo Antonelli

Policlinico Universitario Agostino Gemelli IRCCS 
Research

Keywords: Tigecycline, high dose, pharmacokinetics, epithelial lining fluid, critically ill patients, severe infections

Posted Date: February 28th, 2020

DOI: https://doi.org/10.21203/rs.3.rs-15408/v1

License: (c) (i) This work is licensed under a Creative Commons Attribution 4.0 International License.

Read Full License 


\section{Abstract}

\section{Background}

In critically ill patients, the use of high tigecycline dosages (HD TGC) (200 mg/day) has been recently increasing but few pharmacokinetic/pharmacodynamic (PK/PD) data are available. We investigated plasmatic and pulmonary concentrations of HD TGC in the treatment of severe infections.

\section{Methods}

This was a single centre, prospective, observational study that was conducted in the twenty-bed mixed ICU of a 1,500- bed teaching hospital in Rome, Italy. In all patients admitted to the ICU between 2015 and 2018, who received TGC (200 mg loading dose, then $100 \mathrm{mg} \mathrm{q12}$ ) for the treatment of documented infections, serial blood samples were collected to measure TGC concentrations. Moreover, epithelial lining fluid (ELF) concentrations were determined in patients with nosocomial pneumonia.

\section{Results}

Among the 32 non-obese patients included, 11 had a treatment failure, while the other 21 subjects successfully eradicated the infection. There were no between-group differences in terms of demographic aspects and main comorbidities. In nosocomial pneumonia, for a target AUC0-24/MIC of $4.5,75 \%$ of the patients would be successfully treated in presence of $0.5 \mathrm{mg} / \mathrm{L} \mathrm{MIC}$ value and all the patients obtained the PK target with MIC $\leq 0.12 \mathrm{mg} / \mathrm{mL}$. In intra-abdominal infections, for a target AUC0-24/MIC of 6.96, at least $50 \%$ of the patients would be adequately treated against bacteria with $\mathrm{MIC} \leq 0.5 \mathrm{mcg} / \mathrm{mL}$. Finally, in skin and soft-tissue infections, for a target AUC0-24/MIC of $17 ., 9$ only $25 \%$ of the patients obtained the PK target at MIC values of $0.5 \mathrm{mg} / \mathrm{L}$ and less than $10 \%$ were adequately treated against germs with MIC value $\geq 1 \mathrm{mcg} / \mathrm{ml}$. HD TGC showed a relevant pulmonary penetration with a median and IQR ELF/plasma ratio $(\%)$ of 152.9 [73.5-386.8].

\section{Conclusions}

The use of HD TGC is associated with satisfactory plasmatic and pulmonary concentrations for the treatment of severe infections due to fully susceptible bacteria (MIC $<0.5 \mathrm{mg} / \mathrm{L}$ ). Even higher dosages and combination strategies are required in presence of difficult to treat pathogens.

\section{Background}

Tigecycline (TGC), the first antimicrobial of glycilcycline class, has shown an expanded-spectrum activity against gram-positive, gram-negative, aerobic, anaerobic and atypical bacterial species, including antibiotic-resistant strains [1]. Indeed, it has been demonstrated that methicillin-resistant Staphylococcus aureus (MRSA), penicillin- resistant Streptococcus pneumoniae, vancomycin-resistant enterococci (VRE), extended-spectrum $\beta$-lactamase (ESBL)-producing Gram-negative bacilli and extensively drug-resistant (XDR) Acinetobacter baumannii are susceptible to TGC [2-4]. 
TGC is currently approved by the U.S. food and Drug Administration (FDA) for complicated skin and skinstructure infections, complicated intra-abdominal infections, community-acquired pneumonia with an initial dose of $100 \mathrm{mg}$, followed by $50 \mathrm{mg}$ every 12 hours. Nevertheless, due to an increased risk of death compared to other antimicrobials, its use has recently been restricted in situations when alternative treatments are not suitable [5].

However, the alarming increase in antimicrobial resistance among the nosocomial pathogens is leading the clinicians to consider the use of TGC as an important therapy in the management of difficult to treat infection, particularly in critically ill patients. This is also supported by recent studies suggesting that previous failures of TGC therapy in critically ill patients were likely due to a drug underdosage $[6,7]$ and that standard doses provide serum concentrations that are below the minimum-inhibitory concentrations (MICs) of most MDR pathogens. Moreover, it has been reported an increased effectiveness of high-dose TGC (HD TGC) regimen to improve the clinical outcome, without safety issues [8-11].

Therefore, we designed this prospective observational study to describe the pharmacokinetic/pharmacodynamic (PK/PD) profile of HD TGC in a cohort of critically ill patients with severe infections.

\section{Methods}

\section{Patients and study design}

This was a prospective, observational study that was performed between 2015 and 2018 in the 20-bed ICU of a 1,500- bed teaching hospital in Rome, Italy. The protocol was approved by the Catholic University's Ethical Committee (approval number Prot.sf 8431/13). Written informed consent was obtained from the patients' legally authorized representative. Critically ill adult patients were considered eligible for the study when the attending physician prescribed TGC as empirical treatment (within $12 \mathrm{~h}$ from microbiological sampling) of a possible MDR infection, or as targeted therapy based on definitive results, in the absence of any exclusion criteria: known TGC allergy, creatinine clearance less than $40 \mathrm{~mL} / \mathrm{min}$ (calculated according to the Cocrockft-Gault formula) apart from those ones who were anuric and on continuous renal replacement therapy (CRRT), hyperbilirubinemia (bilirubin level higher than $3 \mathrm{mg} / \mathrm{dL}$ ), severe hepatic failure (Child-Pugh C), little chance of survival as defined by the Simplified Acute Physiology 2 (SAPS 2) score $>80$, concomitant treatment with other drugs that can potentially interfere with TGC. TGC was administered intravenously at loading dose (LD) of $200 \mathrm{mg}$ over 30-min, followed by $100 \mathrm{mg}$ over 30-min bid. On day four after the commencement of the HD TGC, at steady state, pharmacokinetic analyses of the study group were performed. Clinical and demographic data were recorded upon enrolment. Safety and adverse events were determined through the observed biochemical abnormalities, documented according to the Department of Health and Human Services-Common Terminology Criteria for Adverse Events (DHHS-CTCAE v.3.0) classification [12]. 
Clinical cure was defined as the complete resolution of all signs and symptoms of the infection by the end of TGC therapy. In case of ventilator associated pneumonia (VAP), improvement or lack of progression of all abnormalities on chest radiographs was also required [13]. Clinical outcomes were independently evaluated by two physicians (GDP, MSV) who were blinded to the treatment. When judgments were discordant (about $5 \%$ of patients), the reviewers reassessed the data and reached a consensus decision. Adequate source control included drainage of infected fluid collections, debridement of infected solid tissue, removal of devices/foreign bodies, and definitive measures to correct anatomic derangements resulting in on-going microbial contamination and to restore optimal function within $48 \mathrm{~h}$ after diagnosis [14].

\section{Sample collection}

Blood samples were collected after the seventh dose (on day 4 of treatment) at T0 (immediately before the initiation of the infusion) and 1, 1.5, 2, 4, 6, 8, 10, 12 after the start of infusion. According to patients' respiratory status, one mini-bronchoalveolar lavage (BAL) $(40 \mathrm{~mL}$ sterile $0.9 \%$ saline solution was blindly instilled through a telescopic catheter and immediately aspirated in a trap) was performed at steady state.

\section{Preparation of stock solution and calibration standard}

Stock solution of TGC and the internal standard (IS), propranolol hydrochloride, were prepared by dissolving accurately-weighed amounts of each compound in $\mathrm{MeOH}$ to obtain a final concentration of $0.1 \mathrm{mg} / \mathrm{ml}$. Calibration standards were prepared by diluting stock solutions of TGC in drug-free human plasma to yield TGC concentrations of $5000,2500,1250,625,312.5,156.25,78.125,39.1,19.5$ and $9.76 \mathrm{ng} / \mathrm{ml}$.

\section{Sample preparation}

\section{Tigecycline liquid/liquid extraction from plasma samples}

Liquid/liquid extraction was employed for purification and concentration of TGC from plasma samples. Briefly $25 \mu \mathrm{l}$ of $0.1 \mathrm{mg} / \mathrm{ml} \mathrm{IS}$ and then $50 \mu \mathrm{l}$ of mobile phase $\mathrm{A}\left(\mathrm{H}_{2} \mathrm{O} 0.1 \% \mathrm{HCOOH}\right)$ were added to $250 \mu \mathrm{l}$ of the plasma sample. After a rapid vortex mixing, $2.5 \mathrm{ml}$ of Methyl-tert butyl ether was added to all samples, the mixture was vortexed for $5 \mathrm{~min}$ and then centrifuged at $3200 \mathrm{rpm}$ for $10 \mathrm{~min}$ at $10^{\circ} \mathrm{C}$. The upper organic layer was separated and evaporated to dryness at $40{ }^{\circ} \mathrm{C}$. The residue was then reconstituted in $100 \mu \mathrm{l}$ of mobile phase $\mathrm{B}[\mathrm{ACN}(+0.1 \% \mathrm{HCOOH})]$ solution and transferred to an autosampler vial.

\section{Tigecycline solid-phase extraction from BAL samples}

Purification and concentration of TGC from BAL samples were carried out by solid-phase extraction (SPE). Briefly, $500 \mu \mathrm{L}$ of BAL samples were mixed to $500 \mu \mathrm{L}$ of $1 \%$ TFA in water, and $6 \mu \mathrm{L}$ of concentrated TFA were subsequently added. After 5 min of vortex, the samples were centrifuged at $2500 \mathrm{rpm}$ for 10 min; thereafter, the surnatants were collected and loaded onto SPE cartridges (OASIS HLB 1 cc; 
Waters) arranged on an extraction manifold (Vac-Elut, Analytichem International). The SPE cartridges were sequentially pre-conditioned with $1 \mathrm{ml}$ of $\mathrm{MeOH}$ given twice, and subsequently with $1 \mathrm{ml}$ of water given twice. The loaded cartridges were washed with $1 \mathrm{ml}$ of $1 \%$ TFA in water, and eluted with $1 \mathrm{ml}$ of $\mathrm{MEOH}$. The eluted solutions were evaporated to dryness at $40{ }^{\circ} \mathrm{C}$. The residues were reconstituted in $100 \mu \mathrm{l}$ of solution and then transferred to an auto-sampler vial

\section{Chromatographic and Mass-Spectrometric Conditions}

The chromatographic separation was performed on an AQUITY UPLC system (Waters Corp., Milford, MA, USA) with cooled auto-sampler and column oven for temperature control. An AQUITY UPLC BEH C18 column (1.7 um; $2.1 \times 50 \mathrm{~mm}$; Waters Corp., Milford, MA, USA) was employed and the column temperature was maintained at $40{ }^{\circ} \mathrm{C}$. The elution was performed at a flow rate of $0.6 \mathrm{~mL} / \mathrm{min}$ with a run time of $3 \mathrm{~min}$. The UPLC was connected to a triple quadrupole tandem mass detector (TQD) (Waters Corp., Milford, MA, USA) with an electrospray ionization (ESI) source for the mass spectrometric detection. The ESI source was set in positive ion mode and the quantification was performed using multi reaction monitoring (MRM) mode for the most suitable mass transitions. The optimal mass spectroscopy (MS) parameters were showed in Table 1. Data were processed using MassLynx software with a QuanLynx program version 4.1 (Waters Corp., Milford, MA, USA). 
Table 1

Baseline patients characteristics

$\begin{array}{llll}\text { Total cohort } & \text { Treatment } & \text { Treatment } & p \\ (n=32) & \text { Failure } & \text { Success } & \text { value } \\ & (n=11) & (n=21) & \end{array}$

Demographics and comorbidities

\begin{tabular}{|lllll|}
\hline Age, years & $56[46-68.5]$ & $55[49.75-71]$ & $56[45-68.25]$ & 0.75 \\
\hline Male sex, N (\%) & $17(53.1)$ & $5(45.5)$ & $12(57.1)$ & 0.8 \\
\hline Weight, $(\mathrm{Kg})$ & $76.5[60-90]$ & $75[67.8-80]$ & $90[60-100]$ & 0.45 \\
\hline Albumin, (g/dL)* & $23[21.5-26.5]$ & $\begin{array}{l}22[19.25- \\
26.25]\end{array}$ & $24[22.75-26.5]$ & 0.17 \\
\hline Fluid balance, (mL)* & $+762.9[-393-$ & $+3332[-1124.2-$ & $616.3[-358.5-$ & 0.5 \\
\hline SAPS II score & $+3703.5]$ & $+4112]$ & $+2592.7]$ & \\
\hline Cardiovascular diseases, N (\%) & $6(18.75)$ & $3(27.3)$ & $32[43.5-67.5]$ & 0.92 \\
\hline COPD, N (\%) & $5(15.6)$ & $1(9.1)$ & $4(14.3)$ & 0.39 \\
\hline Chronic renal failure, N (\%) & $7(21.9)$ & $3(27.3)$ & $4(19.1)$ & 0.64 \\
\hline Diabetes, N (\%) & $3(9.4)$ & 0 & $3(14.3)$ & 0.4 \\
\hline Neoplasm, N (\%) & $7(21.9)$ & $4(36.4)$ & $3(14.3)$ & 0.2 \\
\hline Presenting features and outcomes & & & & 0.53 \\
\hline ICU LOS before TGC, (days) & $7.5[2.5-16]$ & $5[0.5-11.25]$ & $12[3.75-18.25]$ & 0.13 \\
\hline MV duration before TGC (days) & $8[3-12]$ & $5[0.5-11.25]$ & $8[3.75-14.75]$ & 0.19 \\
\hline $\begin{array}{l}\text { Vasopressors duration before } \\
\text { TGC (days) }\end{array}$ & $4.5[0-8.5]$ & $5[0.25-8.25]$ & $4[0-8.25]$ & 0.89 \\
\hline
\end{tabular}

Data are presented as median [IQR], unless otherwise indicated

Pts: patients; VAP: ventilator-associated pneumonia; TGC: tigecycline; SAPS II: Simplified Acute Physiology Score; COPD: chronic obstructive pulmonary disease; LOS: length of stay; ICU: Intensive Care Unit; MV: mechanical ventilation; SOFA: Sequential Organ Failure Assessment; AKI: acute kidney injury; CRRT: continuous renal replacement therapy; ARF: acute respiratory failure; MV: mechanical ventilation; Kg: kilogramsIQR: interquartile range

* Evaluated at TGC starting day

** i.e. Sreptococcus spp., staphylococcus aureus, enterococci

*** i.e. Acinetobacter baumannii, Klebsiella pneumonia, Enterobacteriaceae, Bacteroides spp. 


\begin{tabular}{|c|c|c|c|c|}
\hline & $\begin{array}{l}\text { Total cohort } \\
(n=32)\end{array}$ & $\begin{array}{l}\text { Treatment } \\
\text { Failure } \\
(n=11)\end{array}$ & $\begin{array}{l}\text { Treatment } \\
\text { Success } \\
(n=21)\end{array}$ & $\begin{array}{l}\mathrm{p} \\
\text { value }\end{array}$ \\
\hline SOFA score* & $7[4-10]$ & 8 [4.75-12] & $6[4-9]$ & 0.2 \\
\hline Septic shock, N (\%)* & $18(56.3)$ & $7(63.6)$ & $11(52.4)$ & 0.71 \\
\hline ARF requiring $\mathrm{MV}, \mathrm{N}(\%)^{*}$ & $28(87.5)$ & $10(90.9)$ & $18(85.7)$ & 1 \\
\hline AKI requiring CRRT, $\mathrm{N}(\%)^{*}$ & $11(34.4)$ & $3(27.3)$ & $8(38.1)$ & 0.7 \\
\hline Creatinine clearance $(\mathrm{ml} / \mathrm{min})^{\star}$ & $97.3[32-150.8]$ & $63.2[32-155]$ & $104[30-142]$ & 0.85 \\
\hline VAP, N (\%) & $19(59.4)$ & $3(27.3)$ & $16(76.2)$ & 0.02 \\
\hline $\begin{array}{l}\text { Intra-abdominal infection, } \mathrm{N} \\
(\%)\end{array}$ & $10(31.3)$ & $6(54.5)$ & $4(19.1)$ & 0.06 \\
\hline $\begin{array}{l}\text { Skin and soft-tisuue infection, } \\
\mathrm{N}(\%)\end{array}$ & $3(9.4)$ & $2(18.2)$ & $1(4.8)$ & 0.27 \\
\hline Secundary bacteraemia, N (\%) & $13(40.6)$ & $4(36.4)$ & $9(42.9)$ & 1 \\
\hline Source control, N (\%) & $13(40.6)$ & $7(63.6)$ & $6(28.6)$ & 0.07 \\
\hline TGC therapy duration,(days) & $12[9-15]$ & $12[10-15]$ & $11[8-17]$ & 0.69 \\
\hline TGC empirical therapy, $\mathrm{N}(\%)$ & $17(53.1)$ & $7(63.6)$ & $10(47.6)$ & 0.47 \\
\hline 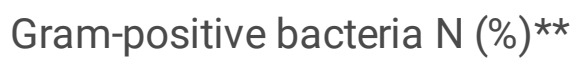 & $11(34.4)$ & $4(36.4)$ & 7 (33.3) & 1 \\
\hline $\begin{array}{l}\text { Gram-negative bacteria } \mathrm{N} \\
(\%)^{\star \star \star}\end{array}$ & $29(90.6)$ & $10(90.9)$ & $19(90.5)$ & 1 \\
\hline ICU LOS after TGC, (days) & 15 [10.5-27] & $14.5[12-19]$ & $16[10-31.4]$ & 0.42 \\
\hline MV duration after TGC (days) & $10[5-15]$ & 14 [9.75-15.75] & $8[2-13.5]$ & 0.04 \\
\hline $\begin{array}{l}\text { Vasopressors duration after } \\
\text { TGC (days) }\end{array}$ & $3[1.5-13]$ & 8 [2.25-13] & 3 [0-10.75] & 0.12 \\
\hline
\end{tabular}

Data are presented as median [IQR], unless otherwise indicated

Pts: patients; VAP: ventilator-associated pneumonia; TGC: tigecycline; SAPS II: Simplified Acute Physiology Score; COPD: chronic obstructive pulmonary disease; LOS: length of stay; ICU: Intensive Care Unit; MV: mechanical ventilation; SOFA: Sequential Organ Failure Assessment; AKI: acute kidney injury; CRRT: continuous renal replacement therapy; ARF: acute respiratory failure; MV: mechanical ventilation; Kg: kilogramsIQR: interquartile range

* Evaluated at TGC starting day

** i.e. Sreptococcus spp., staphylococcus aureus, enterococci

*** i.e. Acinetobacter baumannii, Klebsiella pneumonia, Enterobacteriaceae, Bacteroides spp. 


\begin{tabular}{|c|c|c|c|c|}
\hline & $\begin{array}{l}\text { Total cohort } \\
(n=32)\end{array}$ & $\begin{array}{l}\text { Treatment } \\
\text { Failure } \\
(n=11)\end{array}$ & $\begin{array}{l}\text { Treatment } \\
\text { Success } \\
(\mathrm{n}=21)\end{array}$ & $\begin{array}{l}\mathrm{p} \\
\text { value }\end{array}$ \\
\hline 30-day mortality & $9(28.1)$ & $8(72.7)$ & $1(4.8)$ & $\stackrel{<}{0.001}$ \\
\hline \multicolumn{5}{|c|}{ Data are presented as median [IQR], unless otherwise indicated } \\
\hline \multicolumn{5}{|c|}{$\begin{array}{l}\text { Pts: patients; VAP: ventilator-associated pneumonia; TGC: tigecycline; SAPS II: Simplified Acute } \\
\text { Physiology Score; COPD: chronic obstructive pulmonary disease; LOS: length of stay; ICU: Intensive } \\
\text { Care Unit; MV: mechanical ventilation; SOFA: Sequential Organ Failure Assessment; AKI: acute kidney } \\
\text { injury; CRRT: continuous renal replacement therapy; ARF: acute respiratory failure; MV: mechanical } \\
\text { ventilation; Kg: kilogramsIQR: interquartile range }\end{array}$} \\
\hline \multicolumn{5}{|c|}{ * Evaluated at TGC starting day } \\
\hline \multicolumn{5}{|c|}{ ** i.e. Sreptococcus spp., staphylococcus aureus, enterococci } \\
\hline
\end{tabular}

\section{Urea assay}

\section{Determination of urea in plasma and BAL samples}

Urea levels were detected by the QuantiChrom Urea Assay kit (BIOassay System, Hayward, CA, USA), which was used according to the manufacturer's instructions.

\section{Pharmacokinetic/pharmacodynamics analysis}

A one compartment model with first-order elimination determined pharmacokinetic parameters. The $0-$ $12 \mathrm{~h}$ area under the time concentration curve $\left(\mathrm{AUC}_{0-12}\right)$ was determined by the linear trapezoidal rule. $\mathrm{TGC} \mathrm{AUC}_{0-24}$ was calculated as $\mathrm{AUC}_{0-12} \times 2$. TGC maximum and minimum concentrations (Cmax, Cmin) were directly obtained from observed peak and trough concentrations. Epithelial lining fluid (ELF) tigecycline $\left(\mathrm{TGC}_{\mathrm{ELF}}\right)$ concentration was calculated from $\mathrm{BAL}$ concentration $\left(\mathrm{TGC}_{\mathrm{BAL}}\right)$ using urea as dilution marker: $\mathrm{TGC}_{\mathrm{ELF}}=\mathrm{TGC}_{\mathrm{BAL}} \mathrm{X}$ urea dilution index (plasma urea concentration/BAL urea concentration) [15]. In all patients, distribution volume (Vd), drug clearance (CL), and elimination half-life $\left(t_{1 / 2}\right)$ were calculated after a single 100-mg intravenous dose at steady state.

Area under the concentration curve (AUC) ${ }_{0-24} / \mathrm{MIC}$ ratio $\geq 4.5,6.96$ and 17.9 were used as PD targets for VAP, intra-abdominal infections (IAI) and skin-soft tissue infections (SSTI), respectively [6]. Graphing of data was undertaken using Prism version 6.0 for Windows (graphPad Software, San Diego, CA).

\section{Microbiological analysis}

Isolates were identified by matrix-assisted laser desorption ionization-time-of-flight (MALDI-TOF) mass spectrometry (MALDI Biotyper, Bruker Daltonics GmbH, Leipzig, Germany). The in vitro susceptibility of 
the isolates was assessed with the Vitek 2 system (bioMérieux, Marcy l'Etoile, France) or with panels manufactured by MERLIN Diagnostica GmbH (Bornheim, Germany). Results were interpreted in accordance with the European Committee on Antimicrobial Susceptibility Testing (EUCAST) clinical breakpoints. The presence of carbapenemase genes of blaKPC, blaNDM, blaVIM, blaOXA-48, blaOXA-23, and blaOXA-58 types was determined by polymerase chain reaction and DNA sequencing analysis using previously described protocols (Endemiani, Poirel, Woodford). [16]

\section{Statistical analysis}

All statistical analyses were performed using MedCalc software, version 12.2.1 (MedCalc $\circledast$, MariaKerke, Belgium). Kolmogorov-Smirnov test was used to value the variables distribution. The data with a nonNormal distribution were assessed with Mann-Whitney test and the median and selected centiles (25th75th) value was given (interquartile range, IQR). The data with a Normal distribution were assessed with Student's test. Categorical variables are presented as proportions and were analysed with the use of the chi-square test or Fisher's exact test, as appropriate. A P value $<0.05$ was considered significant. Due to the PK/PD design of the study, a sample size was not calculated, foreseeing the recruitment of at least 30 patients during the predefined study period (July 2015-July 2017).

\section{Results}

\section{Patients characteristics}

The clinical details of the 32 non-obese patients included in the study are listed in Table 1. Albumin levels were quite low with an overall positive fluid balance at enrolment. Median SAPS II score was 53.5 and the most relevant comorbidities were cardiovascular diseases, chronic obstructive pulmonary disease, chronic renal failure and neoplasm (Table 1). Median SOFA score was 7 and many patients were in septic shock or presented with acute respiratory failure (ARF) and acute kidney injury (AKI) requiring mechanical ventilation (MV) and CRRT, respectively. More than half of the patients had VAP, followed by intraabdominal infections and skin and soft-tissue infections: in the microbiological case-mix Gram-negative bacteria were mostly represented (90.6\%). Median duration of TGC therapy was 12 days and it was started empirically in half of the cases. The use of vasopressors and MV during TGC therapy was high and 30 -day mortality rate was $28.1 \%$. Eleven patients had a treatment failure, while the other 21 successfully eradicated the infection. There were no between-group differences in terms of demographic aspects and main comorbidities. Further the two groups were similar in terms of presenting features and outcomes with the exception of VAP rate which was higher in the treatment success group $(76.2 \%$ vs. $27.3 \%, p=0.02$ ) and a trend to a higher percentage of skin and soft tissue infections and source control among patients who failed TGC treatment ( $p=0.06$ and $p=0.07$, respectively).

\section{Pharmacokinetic results}

A one-compartment model with first-order disposition processes adequately described the concentrationtime curve, although significant inter-individual variability was observed. $\mathrm{Vd}, \mathrm{Cl}$ and $\mathrm{t}_{1 / 2}$ were $438.6 \mathrm{~L}$, 
42.1 L/h and 7.2 h, respectively. Median and IQR values of Cmax and Cmin were 0.34 [0.15-1.03] mg/L and $0.09[0.05-0.26] \mathrm{mg} / \mathrm{L}$ (Table 1). Figure 1 shows the mean $\pm S D$ time-concentration profile at different time points of plasma tigecycline concentrations, compared with most frequently observed MIC values $(0.12-0.25-0.5 \mathrm{mcg} / \mathrm{mL}) . \mathrm{AUC}_{0-24}$ and IQR was calculated for each patient and the percentage of target

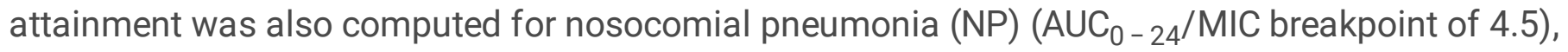
complicated intra-abdominal infections (CIAI) $\left(\mathrm{AUC}_{0-24} / \mathrm{MIC}\right.$ breakpoint of 6.96) and complicated skin and soft tissue infections (CSSTI) $\left(\mathrm{AUC}_{0-24} / \mathrm{MIC}\right.$ breakpoint of 17.9). Considering a target $\mathrm{AUC}_{0-24} / \mathrm{MIC}$ of $4.5,75 \%$ of the patients would be successfully treated in presence of $0.5 \mathrm{mg} / \mathrm{L}$ MIC value and all the patients obtained the PK target with $\mathrm{MIC} \leq 0.12 \mathrm{mg} / \mathrm{mL}$. Considering a target $\mathrm{AUC}_{0-24} / \mathrm{MIC}$ of 6.96 , at least $50 \%$ of the patients would be adequately treated against bugs with MIC $\leq 0.5 \mathrm{mcg} / \mathrm{mL}$, while only $15.6 \%$ obtained the PK target with MIC of $2 \mathrm{mg} / \mathrm{mL}$. Finally, with a target $\mathrm{AUC}_{0-24} / \mathrm{MIC}$ of 17.9 only $25 \%$ of the patients obtained the PK target at MIC values of $0.5 \mathrm{mg} / \mathrm{L}$ and less than $10 \%$ were adequately treated against germs with MIC value $\geq 1 \mathrm{mcg} / \mathrm{ml}$ (Fig. 2).

\section{Pulmonary concentrations}

Tigecycline pulmonary concentrations were measured in $12(1 \mathrm{~h})$ and $7(12 \mathrm{~h})$ patients, respectively. Main reasons to exclude samples were presence of blood and excessive dilution, while five patients did not undergo BAL due to severe respiratory failure. Median and IQR ELF Cmax was 0.42 [0.15-1.2] and ELF Cmin was 0.32 [0.17-0.43]; median and IQR ELF/plasma ratio (\%) was 152.9 [73.5-386.8] (Table 2). Mean $\pm S E$ ELF concentrations were similar at $1 \mathrm{~h}$ and $12 \mathrm{~h}(0.78 \pm 0.2 \mathrm{mg} / \mathrm{mL}$ vs. $0.36 \pm 0.1 \mathrm{mg} / \mathrm{mL} ; \mathrm{p}=$ 0.19 ) (Fig. 3). Conversely, no significant differences were found comparing mean $\pm S E$ ELF/plasma ratio at $1 \mathrm{~h}$ and $12 \mathrm{~h}(281 \pm 107.6$ vs. $298.3 \pm 60.7 ; \mathrm{p}=0.9)$ (Fig. 4). 
Table 2

Steady-state serum and alveolar TGC PK parameters in the 32 enrolled patients

\section{Parameter}

$\mathrm{Vd}, \mathrm{L}$

$\mathrm{CL}, \mathrm{L} / \mathrm{h}$

$t_{1 / 2}, h$

$\mathrm{C}_{\text {max }}, \mathrm{mg} / \mathrm{L}$

$\mathrm{C}_{\min }, \mathrm{mg} / \mathrm{L}$

$E L F C_{\text {max }}, \mathrm{mg} / \mathrm{L}^{\star}$

$E L F C_{\min }, \mathrm{mg} / \mathrm{L}^{*}$

ELF/plasma ratio (\%), median [IQR]*

$\mathrm{AUC}_{0-24}, \mathrm{mg} \mathrm{h} / \mathrm{L}$

$\mathrm{AUC}_{0-24} / 0.12 \mathrm{mg} / \mathrm{L} \mathrm{MIC} \geq 4.5,(\%)$

$\mathrm{AUC}_{0-24} / 0.25 \mathrm{mg} / \mathrm{L} \mathrm{MIC} \geq 4.5,(\%)$

$\mathrm{AUC}_{0-24} / 0.5 \mathrm{mg} / \mathrm{L} \mathrm{MIC} \geq 4.5,(\%)$

$\mathrm{AUC}_{0-24} / 1 \mathrm{mg} / \mathrm{L} \mathrm{MIC} \geq 4.5$, (\%)

$\mathrm{AUC}_{0-24} / 2 \mathrm{mg} / \mathrm{L} \mathrm{MIC} \geq 4.5,(\%)$

$\mathrm{AUC}_{0-24} / 0.12 \mathrm{mg} / \mathrm{L} \mathrm{MIC} \geq 6.96,(\%)$

$\mathrm{AUC}_{0-24} / 0.25 \mathrm{mg} / \mathrm{L} \mathrm{MIC} \geq 4.5,(\%)$

$\mathrm{AUC}_{0-24} / 0.5 \mathrm{mg} / \mathrm{L} \mathrm{MIC} \geq 6.96,(\%)$

$\mathrm{AUC}_{0-24} / 1 \mathrm{mg} / \mathrm{L} \mathrm{MIC} \geq 6.96,(\%)$
Patients $(n=32)$

438.6

42.1

7.2

$0.34[0.15-1.03]$

$0.09[0.05-0.26]$

$0.42[0.15-1.2]$

$0.32[0.17-0.43]$

$152.9[73.5-386.8]$

$3.61[2.55-10.39]$

100

94

75

40.6

28.1

100

91

50

34.4

Data are expressed as median [IQR] and N (\%)

*TGC ELF concentrations were measured in $12(1 \mathrm{~h})$ and 7 (12 h) samples, respectively

\section{Legend}

TGC tigecycline; PK pharmacokinetic; Vd volume of drug distribution, IQR interquartile range; CL drug clearance; $\mathrm{t}_{1 / 2}$ elimination half-life; $\mathrm{Cmax}$ peak plasmatic concentration; Cmin trough plasmatic concentration; ELF epithelial lining fluid; MIC minimun inhibitory concentration; AUC total drug area under the time-concentration curve 


\section{Parameter}

$$
\mathrm{AUC}_{0-24} / 2 \mathrm{mg} / \mathrm{L} \mathrm{MIC} \geq 6.96,(\%)
$$$$
\mathrm{AUC}_{0-24} / 0.12 \mathrm{mg} / \mathrm{L} \mathrm{MIC} \geq 17.9,(\%)
$$$$
\mathrm{AUC}_{0-24} / 0.25 \mathrm{mg} / \mathrm{L} \mathrm{MIC} \geq 17.9,(\%)
$$$$
\mathrm{AUC}_{0-24} / 0.5 \mathrm{mg} / \mathrm{L} \mathrm{MIC} \geq 17.9 \text {, (\%) }
$$$$
\mathrm{AUC}_{0-24} / 1 \mathrm{mg} / \mathrm{L} \mathrm{MIC} \geq 17.9,(\%)
$$$$
\mathrm{AUC}_{0-24} / 2 \mathrm{mg} / \mathrm{L} \mathrm{MIC} \geq 17.9,(\%)
$$

Data are expressed as median [IQR] and $\mathrm{N}(\%)$

*TGC ELF concentrations were measured in $12(1 \mathrm{~h})$ and $7(12 \mathrm{~h})$ samples, respectively

\section{Legend}

TGC tigecycline; PK pharmacokinetic; Vd volume of drug distribution, IQR interquartile range; CL drug clearance; $\mathrm{t}_{1 / 2}$ elimination half-life; Cmax peak plasmatic concentration; Cmin trough plasmatic concentration; ELF epithelial lining fluid; MIC minimun inhibitory concentration; AUC total drug area under the time-concentration curve

\section{Discussion}

Our study shows a HD TGC (200 mg LD, then $100 \mathrm{mg}$ q12) time-curve concentration with mean peak and trough levels of $0.65 \mathrm{mg} / \mathrm{mL}$ and $0.25 \mathrm{mg} / \mathrm{mL}$, respectively (Fig. 1). AUC ${ }_{0-24} / \mathrm{MIC}$ targets for nosocomial pneumonia ( $\geq 4.5$ ) and complicated intra-abdominal infections $(\geq 6.96)$ were obtained in the majority of cases in presence of bacteria with MIC values $\leq 0.25$. Otherwise lower MIC values $(\leq 0.12 \mathrm{mcg} / \mathrm{mL})$ were required to have satisfactory $\mathrm{AUC}_{0-24} / \mathrm{MIC}$ results $(78 \%)$, while treating a skin/soft tissue infection (Fig. 2, Table 2). Similar to plasma $1 \mathrm{~h}$ and $12 \mathrm{~h}$, pulmonary concentrations $(0.78 \mathrm{mg} / \mathrm{L}$ and $0.36 \mathrm{mg} / \mathrm{L}$, respectively) were observed with a good median ELF/plasma ratio of $152.9 \%$ (Table 2, Fig. 3 ). This highdose regimen was associated with a $65.6 \%$ of treatment success rate in a normal weight population including $60 \%$ of VAP, $31 \%$ of CIAI and $9 \%$ of SSTI. TGC was used in half of the cases as targeted regimen for a median duration of 12 days. The rate of septic shock, acute respiratory failure requiring MV and acute kidney injury requiring CRRT was also high, with a mortality rate of $28.1 \%$ (Table 1).

The pharmacokinetics/pharmacodynamics and tissue penetration of tigecycline have been extensively studied in various in vitro and human models [17]. However, these studies were generally carried out in healthy volunteers, and few pharmacokinetic data concerning infected patients are available, which may present pathophysiologic conditions influencing the pharmacokinetic profile of this molecule. Additionally, the majority of available data in infected patients derive from studies where normal doses are used, although for severe nosocomial infections a double-dose regimen is warranted $[18,19]$ 
Recently, standard dose TGC pharmacokinetics in ten critically ill patients have been studied [6]. The authors observed that a larger body mass index was associated with increased TGC Cl, but standard doses produced satisfactory plasmatic levels for VAP and clAl treatment due to Enterobacter cloacae, Esherichia coli, Klebsiella pneumoniae and methicillin-resistant Staphylococcus aureus. However higher dosages were required for the treatment of SSTI, especially in obese patients.

Eleven out of 32 patients in our cohort were receiving CRRT while being treated with high-dose TGC. Interestingly, in a recent paper, Broeker and cow. [20] described the PK/PD of standard dose TGC in eleven patients on continuous veno-venous hemodialysis (CVVHD) or hemodiafiltration (CVVHDF). TGC dialysability, as expressed by saturation coefficients ( 0.79 and 0.9 for CVVHD and CVVHDF, respectively) was very high, but the contribution of CRRT TGC clearance was minimal (about $2 \mathrm{~L} / \mathrm{h}$ ), compared with the total body clearance $(18.3 \mathrm{~L} / \mathrm{h})$. Peak drug concentrations were below $1 \mathrm{mg} / \mathrm{L}$ and trough levels about $0.2 \mathrm{mg} / \mathrm{L}$. The authors, considering the AUC0-24/MIC referral value for CIAI (6.96), observed that such target was accomplished in $88 \%$ of the case if MIC was $\leq 0.5$.

Indeed, our results are in line with current available data, underlying the plus-value of increased dosages while treating critically ill patients especially with severe cIAI and SSTI. In addition, there is a high need of PK/PD data on TGC administered at higher than approved dosages, in light of the wide spread of increased resistance to TGC among Gram-negative rods and Acinetobacter spp. The first investigation on PK/PD of HD TGC derives from Ramirez et al who conducted a randomized phase 2 trial to evaluate the clinical efficacy of two high-dosage regimen of TGC ( $75 \mathrm{mg}$ bid and $100 \mathrm{mg}$ bid) versus imipenemcilastatin for the treatment of nosocomial pneumonia[8]. In the clinically evaluable population, clinical cure with TGC $100 \mathrm{mg}$ bid was higher than with 75 bid and imipenem-cilastatin ( $85 \%$ vs. $69.6 \%$ vs. $75 \%$ ). Mean peak TGC concentration was about $1 \mathrm{mg} / \mathrm{mL}$, declining to less than $0.5 \mathrm{mg} / \mathrm{ml}$ after 8 hours, observing a safety profile comparable to that one known for the approved those. The only other study investigating the PK/PD of HD TGC profile was conducted by Borsuk-De Moor et al in 37 ICU patients with severe infections [21]. The time-concentration curve was similar to our data, displaying a peak concentration about $1 \mathrm{mg} / \mathrm{L}$ and $12 \mathrm{~h}$ level below $0.5 \mathrm{mg} / \mathrm{L}$. Interestingly, the authors developed a model which showed that no individual covariates may influence target concentrations, advising to modify TGC daily dosage according to pathogens type, susceptibility pattern and PK targets.

Tissue concentrations of antibiotics at the target site contribute to therapeutic effects: using plasma concentrations may frequently overestimate the target site concentrations and therefore clinical efficacy. This is the first study to report steady-state ELF percentage penetration of TGC administered $100 \mathrm{mg} \mathrm{q} 12$ after $200 \mathrm{mg}$ LD. Considering the $\mathrm{AUC}_{0-24} / \mathrm{MIC}$ target of 4.96 , our data shows satisfactory pulmonary concentrations with potential clinical success in $100 \%-94 \%-75 \%-41 \%$ of the cases treating bacteria with MIC of $0.12-0.25 \mathrm{mg} / \mathrm{L}-0.5-1 \mathrm{mg} / \mathrm{L}$, respectively [Fig. 2]. These data confirm the results observed in healthy subjects by Conte et al., where the Cmax/MIC90, AUC/MIC90 ratios, T > MIC90 and extended serum and intrapulmonary half-lives following the standard regimen are favourable for the treatment of TGC-susceptible pulmonary infections [22]. Penetration ratio may be even higher when in presence of infected lungs. Crandon et al. demonstrated in infected and non-infected mice lungs that the baseline 
penetration ratio of 8.1 is incremented to 23.3 in case of Acinetobacter pneumonia [23]. Conversely, the majority of lung penetration occurs in alveolar cells, than in ELF, as suggested by Welte et al. in three cases of MDR lung infections [24]. Finally in a recent study on 58 healthy subjects treated with standard TGC dose, the ratio of ELF and AUC to total plasma concentration of tigecycline was 1.71 and 20.8, respectively [25].

Our study has several limitations. First we adopted a single high-dose of tigecycline and we do not know if even higher dosages may result in better PK/PD profiles. Second we measured only pulmonary tissue concentration trough ELF collection and we can only postulate the real tissue/plasma ratio for clAl and SSTI. Third, our analysis focused on total TGC concentration rather unbound $\mathrm{AUC}_{0-24}$, due to the lack of clinical reliable breakpoint of $\mathrm{fAUC}_{0-24} / \mathrm{MIC}_{90}$. Finally the sample size may be likely responsible of an under-estimated interindividual variability in the observed PK/PD profile.

\section{Conclusions}

Our study is the first investigation where not only plasmatic but also pulmonary tigecycline concentrations are investigated during the treatment of severe infections in critically ill patients with high dose TGC. Observed plasmatic and pulmonary concentrations confirm the safety of this molecule for the treatment of susceptible pathogens, including pneumonia. Higher than $200 \mathrm{mg} /$ day dosages and combination with other active molecules should be adopted while treating Enterobacteriaceae and Acinetobacter spp. with MIC values close to the clinical breakpoint.

\section{Abbreviations}

HD, high dosages; TGC, Tigecycline; PK/PD, pharmacokinetic/pharmacodynamic; ICU, Intensive Care Unit; ELF, epithelial lining fluid; AUC, area under the curve; MIC, minimum inhibitory concentration; IQR, interquartile range; MRSA, Staphylococcus aureus; VRE, vancomycin-resistant enterococci; ESBL, extended-spectrum $\beta$-lactamase; XDR, extensively drug-resistant; FDA, Food and Drug administration; CRRT, continuous renal replacement therapy; SAPS II, Simplified Acute Physiology Score; LD, loading dose; DHHS-CTCAE, Department of Health and Human Services-Common Terminology Criteria for Adverse Events; VAP, ventilator associated pneumonia; BAL, bronchoalveolar lavage; IS, internal standard; SPE, solid-phase extraction; TQD, triple quadrupole tandem; ESI, electrospray ionization; MRM, multi reaction monitoring; MS, mass spectroscopy; Cmax, maximum concentration; Cmin, minimum concentration; Vd, distribution volume; $\mathrm{CL}$, drug clearance; $\mathrm{t1} / 2$, elimination half-life; IAl, intra-abdominal infection; SSTI, skin-soft tissue infection; MALDI-TOF, matrix-assisted laser desorption ionization-time-offlight; EUCAST, European Committee on Antimicrobial Susceptibility Testing; SOFA, Sequential Organ Failure Assessment; ARF, acute respiratory failure; AKI, acute kidney injury; MV, mechanical ventilation; CIAI, complicated intra-abdominal infection; CSSTI, complicated skin and soft tissue infection; CVVHD, continuous veno-venous haemodialysis; CVVHDF, continuous veno-venous hemodiafiltration.

\section{Declarations}


- Ethics approval and consent to participate:The protocol was approved by the Catholic University's Ethical Committee (approval number Prot.sf 8431/13). Written informed consent was obtained from the patients' legally authorized representative.

- Consent for publication: Written informed consent was obtained from the patients' legally authorized representative. No individual person's data in any form are presented in this manuscript.

- Availability of data and materials: The datasets used and/or analysed during the current study are available from the corresponding author on reasonable request.

- Competing interests: The authors declare that they have no competing interests

- Funding: Fondi d'Ateneo 2015 PN

- Authors' contributions: GDP and MA had full access to all the data in the study and take responsibility for the integrity and the accuracy of the data analysis. GDP, MS, PN and MA conceived the study, and participated in its design and coordination and helped to draft the manuscript. GDP was in charge of the statistical analysis, participated in analysis and interpretation of data, helped to draft the manuscript, and critically revised the manuscript for important intellectual content. LL, GMPC, MSV, SLC, LC, CG, GB, LM, SC and VDG collected the data for the study, recruited patients and did sample analyses. GDP, LL, GMPC, MT, MS, PN and MA participated in the conception, design and development of the database, helped in analysis and interpretation of data, helped in drafting of the manuscript and critically revised the manuscript for important intellectual content. All authors read and approved the final manuscript.

- Acknowledgements: None

\section{References}

1. Testa R, Petersen P, Jacobus N, Sum P, Lee V, Tally F: In vitro and in vivo antibacterial activities of the glycylcyclines, a new class of semisynthetic tetracyclines. Antimicrob Agents Chemother 1993, 37(11):2270-2277.

2. Fraise A: Tigecycline: the answer to beta-lactam and fluoroquinolone resistance? J Infect 2006 53(5):293-300.

3. Rodloff A, Dowzicky M: In vitro activity of tigecycline and comparators against a European collection of anaerobes collected as part of the Tigecycline Evaluation and Surveillance Trial (T.E.S.T.) 20102016. Anaerobe 2018, 51:78-88.

4. Nørskov-Lauritsen N, Marchandin H, Dowzicky M: Antimicrobial susceptibility of tigecycline and comparators against bacterial isolates collected as part of the TEST study in Europe (2004-2007). Int J Antimicrob Agents 2009, 34(2):121-130.

5. FDA: FDA Drug Safety Communication: FDA warns of increased risk of death with IV antibacterial Tygacil (tigecycline) and approves new Boxed Warning.

6. Xie J, Wang T, Sun J, Chen S, Cai J, Zhang W, Dong H, Hu S, Zhang D, Wang X et al: Optimal tigecycline dosage regimen is urgently needed: results from a pharmacokinetic/pharmacodynamic 
analysis of tigecycline by Monte Carlo simulation. Int J Infect Dis 2014, 18:62-67.

7. Stein G, Babinchak T: Tigecycline: an update. Diagn Microbiol Infect Dis 2013, 75(4):331-336.

8. Ramirez J, Dartois N, Gandjini H, Yan J, Korth-Bradley J, McGovern P: Randomized phase 2 trial to evaluate the clinical efficacy of two high-dosage tigecycline regimens versus imipenem-cilastatin for treatment of hospital-acquired pneumonia. Antimicrob Agents Chemother 2013, 57(4):1756-1762.

9. De Pascale G, Montini L, Pennisi M, Bernini V, Maviglia R, Bello G, Spanu T, Tumbarello M, Antonelli M: High dose tigecycline in critically ill patients with severe infections due to multidrug-resistant bacteria. Crit Care 2014, 18(3):R90.

10. Humphries R, Kelesidis T, Bard JD, Ward K, Bhattacharya D, Lewinski M: Successful treatment of panresistant Klebsiella pneumoniae pneumonia and bacteraemia with a combination of high-dose tigecycline and colistin. J Med Microbio/ 2010, 59(Pt 11):1383-1386.

11. Falagas M, Vardakas K, Tsiveriotis K, Triarides N, Tansarli G: Effectiveness and safety of high-dose tigecycline-containing regimens for the treatment of severe bacterial infections. Int $J$ Antimicrob Agents 2014, 44(1):1-7.

12. Department of Health and Human Services: Cancer therapy evaluation program, common terminology criteria for adverse events, v.3.0.

13. Niederman M, Chastre J, Corkery K, Fink J, Luyt C, García M: BAY41-6551 achieves bactericidal tracheal aspirate amikacin concentrations in mechanically ventilated patients with Gram-negative pneumonia. Intensive Care Med 2012, 38(2):263-271.

14. De Pascale G, Carelli S, Vallecoccia M, Cutuli S, Taccheri T, Montini L, Bello G, Spanu T, Tumbarello M, Cicchetti A et al: Risk factors for mortality and cost implications of complicated intra-abdominal infections in critically ill patients. J Crit Care 2019, 50:169-176.

15. Boselli E, Breilh D, Rimmelé T, Djabarouti S, Toutain J, Chassard D, Saux M, Allaouchiche B: Pharmacokinetics and intrapulmonary concentrations of linezolid administered to critically ill patients with ventilator-associated pneumonia. Crit Care Med 2005, 33(7):1529-1533.

16. Rasheed J, Tenover F: Detection and Characterization of Antimicrobial Resistance Genes in Pathogenic Bacteria *. Versalovic J, Carroll K, Funke G, Jorgensen J, Landry M, Warnock D (ed), Manual of Clinical Microbiology, 10th Edition ASM Press, Washington, DC 2011:1239-1261.

17. Agwuh K, MacGowan A: Pharmacokinetics and pharmacodynamics of the tetracyclines including glycylcyclines. J Antimicrob Chemother 2006, 58(2):256-265.

18. Geng T, Xu X, Huang M: High-dose tigecycline for the treatment of nosocomial carbapenem-resistant Klebsiella pneumoniae bloodstream infections: A retrospective cohort study. Medicine (Baltimore) 2018, 97(8):e9961.

19. Cunha B, Baron J, Cunha C: Once daily high dose tigecycline - pharmacokinetic/pharmacodynamic based dosing for optimal clinical effectiveness: dosing matters, revisited. Expert Rev Anti Infect Ther 2017, 15(3):257-267.

20. Broeker A, Wicha S, Dorn C, Kratzer A, Schleibinger M, Kees F, Heininger A, Kees M, Häberle H: Tigecycline in critically ill patients on continuous renal replacement therapy: a population 
pharmacokinetic study. Crit Care 2018, 22(1):341.

21. Borsuk-De Moor A, Rypulak E, Potręć B, Piwowarczyk P, Borys M, Sysiak J, Onichimowski D, Raszewski G, Czuczwar M, Wiczling P: Population Pharmacokinetics of High-Dose Tigecycline in Patients with Sepsis or Septic Shock. Antimicrob Agents Chemother 2018, 62(4).

22. Conte JE Jr, Golden J, Kelly M, Zurlinden E: Steady-state serum and intrapulmonary pharmacokinetics and pharmacodynamics of tigecycline. Int $J$ Antimicrob Agents 2005, 25(6):523529.

23. Crandon J, Kim A, Nicolau D: Comparison of tigecycline penetration into the epithelial lining fluid of infected and uninfected murine lungs. $J$ Antimicrob Chemother 2009, 64(4):837-839.

24. Burkhardt O, Rauch K, Kaever V, Hadem J, Kielstein J, Welte T: Tigecycline possibly underdosed for the treatment of pneumonia: a pharmacokinetic viewpoint. Int J Antimicrob Agents 2009, 34(1):101102.

25. Gotfried M, Horn K, Garrity-Ryan L, Villano S, Tzanis E, Chitra S, Manley A, Tanaka S, Rodvold K: Comparison of Omadacycline and Tigecycline Pharmacokinetics in the Plasma, Epithelial Lining Fluid, and Alveolar Cells of Healthy Adult Subjects. Antimicrob Agents Chemother 2017, 61(9).

\section{Figures}
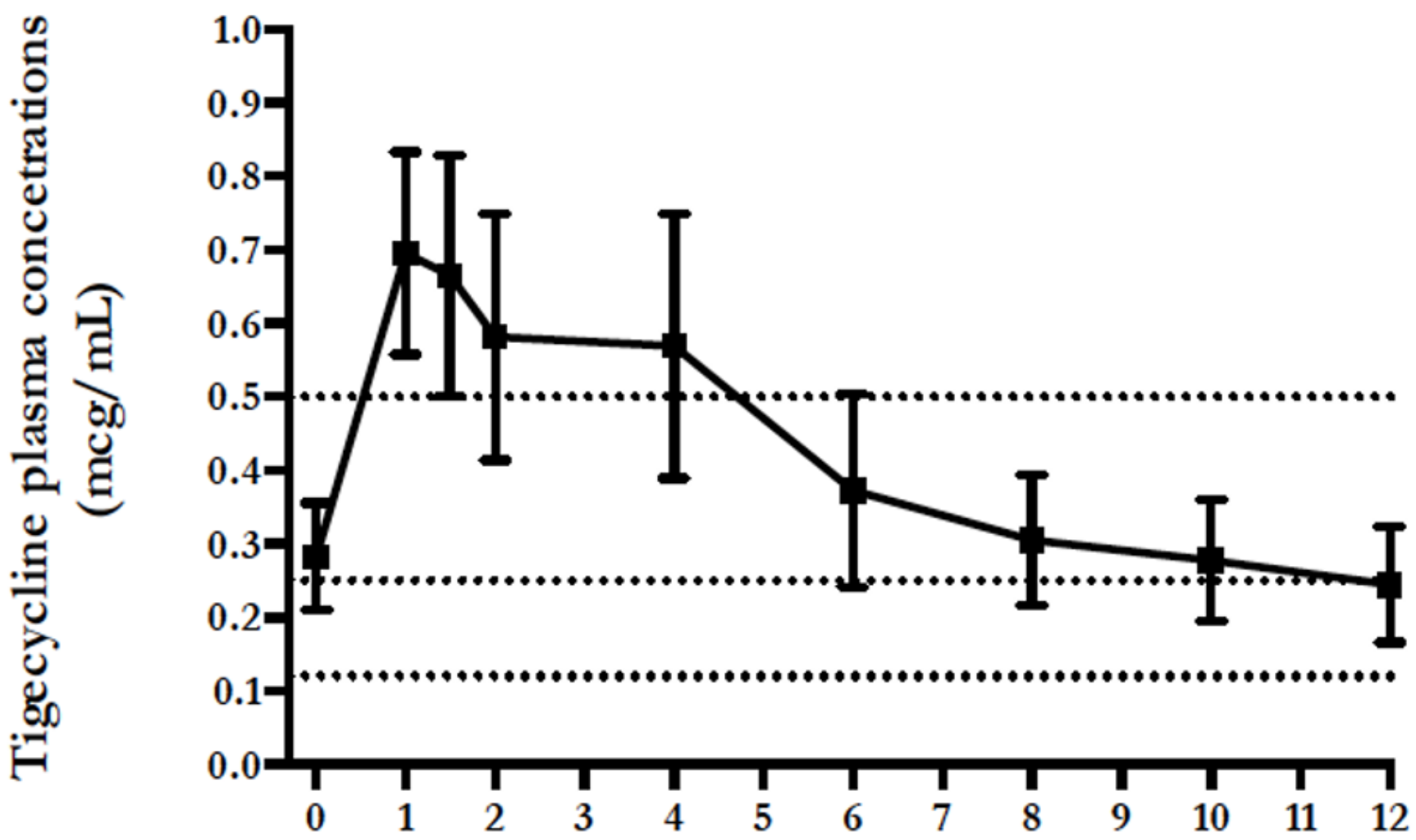

Time (hours) 
Figure 1

Total Tigecycline plasma concentration (mean₫SE) versus time of administration.

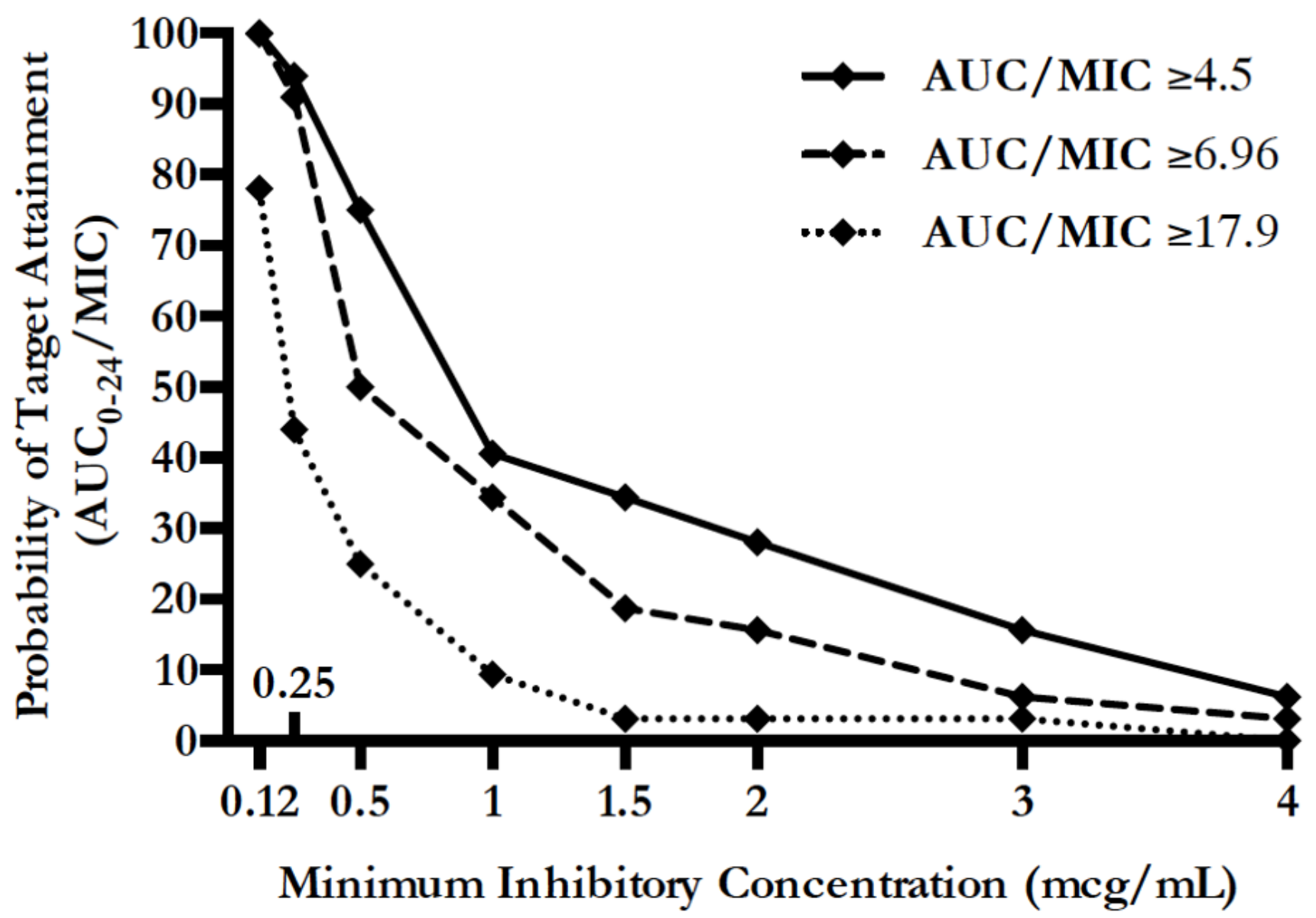

Figure 2

Probability of target attainment of pharmacodynamics indices in plasma, according to different infection types and MIC values 


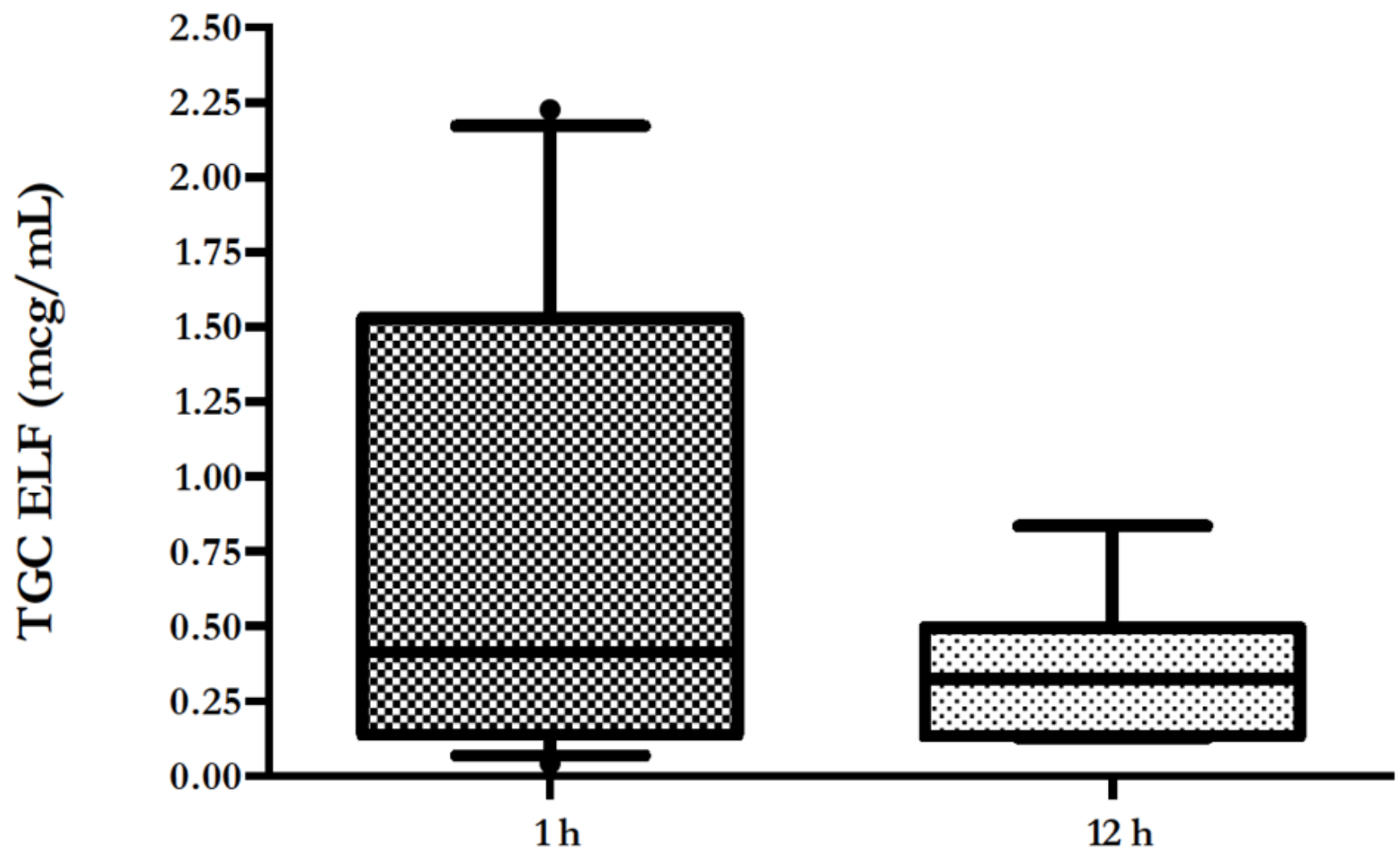

Figure 3

Boxplot showing Tigecycline ELF concentrations. Boxes represent interquartile ranges (lower border 25th percentile; upper border 75th percentile), and the horizontal lines within the boxes indicate the medians (50th percentile). Whiskers indicate minimum and maximum values. 


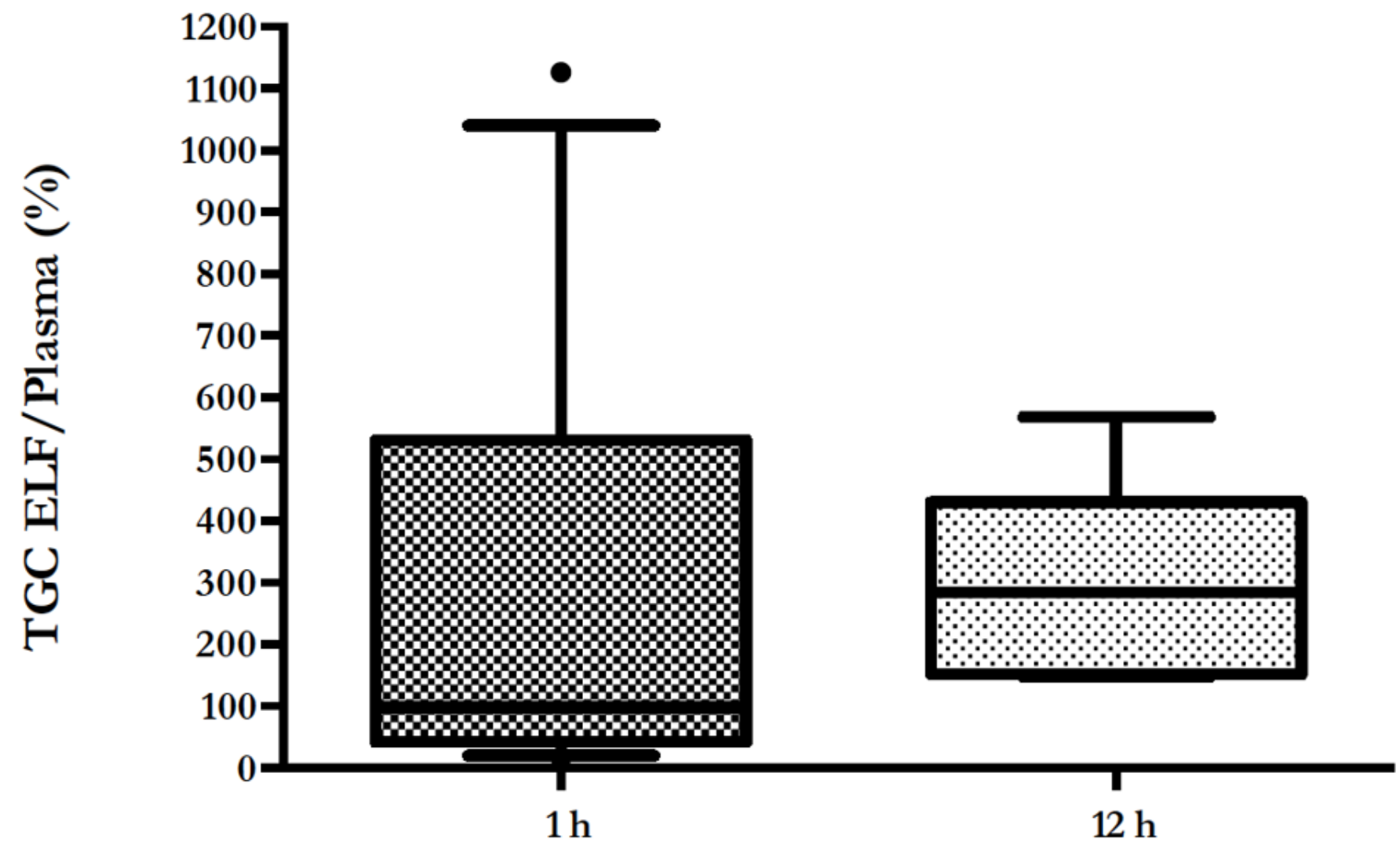

Figure 4

Boxplot showing Tigecycline ELF to plasma ratio (\%). Boxes represent interquartile ranges (lower border 25th percentile; upper border 75th percentile), and the horizontal lines within the boxes indicate the medians (50th percentile). Whiskers indicate minimum and maximum values. 